Revista de Psicología y Educación / Journal of Psychology and Education, 2018, 13(2), $92-98$ (www.rpye.es)

\title{
Deberes escolares y rendimiento académico: un estudio comparativo entre el alumnado inmigrante y nativo
}

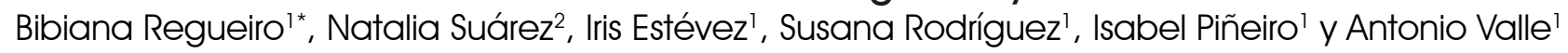

\author{
'Universidad de A Coruña \\ ${ }^{2}$ Universidad de Oviedo
}

\begin{abstract}
Resumen: Los deberes escolares son una de las prácticas educativas más extendidas en todo el mundo. Sin embargo, en literatura previa acerca de esta temática los datos aportados no tienen en cuenta algunas variables importantes y propias de nuestro tiempo, como es la multiculturalidad de las aulas. Así, una variable que podría resultar significativa en la relación entre realizar deberes y rendir en la escuela es el hecho de ser inmigrante o nativo. Por ello, el presente trabajo examina si la relación entre implicación escolar y rendimiento académico varía en función de ésta variable. La muestra se compone de 502 estudiantes (310 nativos y 192 inmigrantes) con edades comprendidas entre los 10 y 16 años participaron en el estudio. A través de la prueba t de Student para muestras independientes los resultados indican que existen diferencias estadísticamente significativas entre las dos poblaciones en la mayoría de las variables estudiadas, a favor del alumnado nativo. Se concluye, por tanto, que los estudiantes nativos aprovechan mejor el tiempo que dedican a la realización de los deberes escolares, llevan al colegio los deberes realizados con una mayor calidad, se benefician más de los mismos y, además, su rendimiento es mayor que los estudiantes inmigrantes.
\end{abstract}

Palabras clave: Inmigrante, Deberes, Calidad de los deberes, Aprovechamiento del tiempo, Rendimiento académico.

\begin{abstract}
Homework and academic achievement: A comparative study between immigrant and native students

Abstract: Homework is one of the greatest educational practices in the world. However, in previous literature about this subject the research contributed does not take into account some important variables, such as the multiculturality of classrooms. Thus, a variable that could be significant in the relationship between doing homework and academic achievement is the fact of being an immigrant or a native. Therefore, the present study examines whether the relationship between homework involvement and academic achievement varies according to this variable. To do this, 502 students (310 natives and 192 immigrants) aged between 10 and 16 participated in the study. Through the Student's t-test for independent samples the results indicate that there are statistically significant differences between the two populations in the majority of the variables studied, in favor of the native students. It is concluded, therefore, that the native students take better advantage of the time they dedicate to do homework, they take to homework realized with a higher quality, they benefit more of homework and, in addition, their academic achievement is better that immigrant students.
\end{abstract}

Keywords: Immigrant, Homework, Quality of homework, Time management, Academic achievement.

En general, los resultados encontrados por la investigación pasada sugieren que los deberes son útiles y necesarios, aunque han de ajustarse a las necesidades del

Recibido: 29/11/2017 - Aceptado: 17/1/2018 - Avance online: 26/1/2018 *Correspondencia: Bibiana Regueiro.

Universidad de A Coruña.

C. P: 15071, A Coruña, España.

E-mail: bibiana.regueiro@udc.es

Regueiro, B., Suárez, N., Estévez, I., Rodríguez, S., Piñeiro, I. y Valle, A. (2018). Deberes escolares y rendimiento académico: un estudio comparativo entre el alumnado inmigrante y nativo Journal of Psychology and Education, 13(2), 92-98, https://dol. $\mathrm{org} / 10.23923 /$ rpye2018.01.160 alumnado (Vila, 2012), así como a los contenidos trabajados en el aula (lbáñez, 2012), no exceder los 10 minutos por curso y día (Cooper, 2007) y realizarse de manera autónoma (Ibáñez, 2012). La gran mayoría de los trabajos realizados sobre este tema han encontrado que aunque la mayor o menor realización de deberes está relacionada significativamente con el mayor o menor

1699-9517/@ 2018 Asociación Científica de Psicología y Educación (ACIPE).

Publicado por Consejo General de Colegios Oficiales de Psicólogos, España. Este es un artículo Open Access bajo la CC BY-NC-ND licencia (http://creativecommons.org/ licencias/by-nc-nd/4.0/). 
rendimiento académico, ésta relación varía en función de la edad y género de los estudiantes (Núñez et al., 2013).

Un problema importante que se observa en la literatura previa acerca de esta temática es que los datos aportados no tienen en cuenta algunas variables importantes y propias de nuestro tiempo, como es la multiculturalidad de las aulas. Dadas las particulares características personales de esta población (lingüísticas, de adaptación, actitudinales, del contexto, etc.) es muy posible que la realización de deberes y su relación con el aprovechamiento escolar sea significativamente diferente que en la población nativa.

Por otro lado y respecto de la implicación en los deberes escolares como tal, algunas publicaciones sugieren una correlación fuerte y positiva entre cantidad de deberes realizados y rendimiento académico (Cooper, 1989; Cooper, Robinson, \& Patall, 2006). Otras destacan que la importancia de realizar las tareas escolares aumenta a medida que los estudiantes avanzan de curso (Zimmerman \& Kitsantas, 2005). Uno de los motivos por los cuales la relación entre deberes y rendimiento es más débil en Primaria que en Secundaria puede ser que las tareas encomendadas por los profesores en los primeros cursos suelen tener como objetivo que los alumnos aprendan a gestionar mejor su tiempo de estudio mediante una mera revisión del material de clase, mientras que el profesorado de Secundaria asigna esos deberes para enriquecer y perfeccionar los conocimientos impartidos en clase (Muhlenbruck, Cooper, Nye, \& Lindsay, 2000).

En relación con la variable tiempo también existe una gran discrepancia, mientras unos encuentran que la relación entre esta variable y el rendimiento es positiva (Cooper, 1989; Trautwein \& Lüdtke, 2009), otros encuentran que es débil o negativa (De Jong, Westerhof, \& Creemers, 2000; Tam, 2009; Trautwein, Schnyder, Niggli, Neumann, \& Lüdtke, 2009). Estos resultados inconsistentes, quizás se expliquen si tenemos en cuenta la tercera variable, el aprovechamiento del tiempo, ya que dedicar más tiempo a los deberes no significa emplear estrategias eficientes de gestión de los mismos ( $\left.X_{u}, 2007\right)$. En investigaciones más recientes como la realizada por Núñez et al. (2013) se concluye que el aprovechamiento del tiempo es la variable de implicación en los deberes que más predice el rendimiento académico.

A tenor de la gran variabilidad de resultados encontrados, Núñez et al. (2013) realizó un estudio en el que además de las tres variables relacionadas con la implicación en los deberes contempló cómo estas se ven relacionadas con la edad y género de los estudiantes. Los resultados indicaron que las chicas hacían más deberes y destinaban más tiempo en labores académicas que los chicos; sin embargo, cabe destacar que no se encontraron diferencias en el aprovechamiento del tiempo. También encontraron que tanto la cantidad de tareas como el aprovechamiento del tiempo destinado a su realización decrecen a medida que aumenta la edad, no encontrándose cambios en función del tiempo dedicado a las tareas.

En el análisis global de las variables que miden el constructo implicación se encontró que la cantidad de deberes se relaciona positivamente con el rendimiento académico, aunque apunta que al igual que sugería Dettmers, Lüdtke, Trautwein, Kunter, \& Baumert (2010) debe controlarse la calidad de los deberes realizados. En relación al tiempo dedicado a los deberes encontraron una relación de doble signo con el rendimiento, negativa cuando se evalúa la relación directa y positiva cuando se tiene en cuenta el aprovechamiento de ese tiempo.

Por lo tanto y a pesar de que los deberes escolares son una de las prácticas educativas más extendidas en todo el mundo, en la literatura previa acerca de esta temática los datos aportados no tienen en cuenta algunas variables importantes y propias de nuestro tiempo, como es la multiculturalidad de las aulas. Así, una variable que podría resultar significativa en la relación entre realizar deberes y rendir en la escuela es el hecho de ser inmigrante o nativo. Por ello, el presente estudio examina si la relación entre implicación escolar y rendimiento académico varía en función de ésta variable. 


\section{MÉTODO}

\section{PARTICIPANTES}

La muestra se compone de 502 estudiantes (310 nativos y 192 inmigrantes) con edades comprendidas entre los 10 y 16 años. De ellos el 85,6\% tiene o procede de una familia de nacionalidad española ( $n=310)$, la otra parte de los escolares, el 14,4\%, está formada por estudiantes inmigrantes o de origen inmigrante $(n=192)$. Casi la totalidad de este alumnado procede de América ( $n=127)$, seguida de inmigrantes de origen europeo $(n=54)$, africano $(n=9)$ y asiático $(n=2)$. Destacar que los grupos de participantes nativos e inmigrantes no difieren estadísticamente en edad y género.

\section{INSTRUMENTOS}

Para medir las variables relativas a los deberes escolares se utilizó la "Encuesta sobre los Deberes Escolares (EDE)" en la que se obtiene información sobre diferentes dimensiones relativas a la eficacia de esta actividad respecto al aprendizaje y al rendimiento académico. La escala ha sido elaborada a partir de otras escalas multi-item publicadas por otros autores en investigaciones previas (e.g., Trautwein, Lüdtke, Schnyder, \& Niggli, 2006; Xu, 2008, 2010), y algunos de los resultados derivados de ésta ya han sido publicados en revistas científicas (e.g., Núñez et al., 2013; Núñez, Suárez, Rosário, Vallejo, Cerezo, \& Valle, 2015; Núñez, Suárez, Rosário, Vallejo, Valle, \& Epstein, 2015).

Para conocer el grado de implicación en los deberes, se recogió información de las siguientes variables: a) número de deberes que realizan habitualmente los alumnos; b) tiempo dedicado a los deberes; y c) aprovechamiento del tiempo dedicado a los deberes. La estimación del número de deberes realizados por los alumnos se obtuvo mediante las respuestas a un ítem relativo a la cantidad de deberes realizados habitualmente, utilizando para ello una escala tipo likert con cinco alternativas ( $1=$ ninguno, 2 =algunos, $3=$ la mitad, 4 = casi todos, 5 = todos).
En cuanto al tiempo diario dedicado a la realización de los deberes, los estudiantes respondieron a tres ítems (en general, en una semana típica, en un fin de semana típico) con la formulación general "¿ Cuánto tiempo sueles dedicar a la realización de los deberes?", siendo las opciones de respuesta $1=$ menos de 30 minutos, 2 = de 30 minutos a una hora, 3 = de una hora a hora y media, $4=$ de hora y media a dos horas, $5=$ más de dos horas.

Finalmente, el aprovechamiento del tiempo dedicado a los deberes se evaluó a través de las respuestas a tres ítems (en general, en una semana típica, en un fin de semana típico) en los que se les pedía que indicaran el nivel de aprovechamiento del tiempo dedicado habitualmente a los deberes, utilizando para ello la siguiente escala: 1 = lo desaprovecho totalmente (me distraigo constantemente con cualquier cosa), 2 = lo desaprovecho más de lo que debiera, 3 = regular, $4=$ lo aprovecho bastante, 5 = lo aprovecho totalmente (me concentro y hasta terminar no pienso en otra cosa). Además de estas variables, se tuvieron en cuenta dos variables más informadas por el profesor: calidad de las tareas para casa realizadas por el alumno y beneficio extraído de su realización, utilizando para ello una escala tipo likert también con cinco alternativas.

Por último, la evaluación del rendimiento académico se obtuvo mediante las calificaciones académicas finales obtenidas en la evaluación final de la asignatura de Matemáticas por los alumnos participantes en el estudio. Se optó por esta materia al ser una de las materias comunes que cursan todos los estudiantes a lo largo de su escolaridad obligatoria.

\section{PROCEDIMIENTO}

Los datos referidos a las variables objeto de estudio fueron recogidos durante el horario escolar por personal externo al propio centro, previo consentimiento del equipo directivo, profesores y padres de los alumnos. Previo a la aplicación de los cuestionarios, que se realizó en un único momento temporal, 
se les informaba a los participantes de la importancia de responder sinceramente a las distintas preguntas planteadas, insistiéndoles que los datos recogidos sólo se iban a utilizar con fines investigadores.

\section{ANÁLISIS DE DATOS}

Teniendo en cuenta los objetivos de este estudio, los datos recogidos han sido analizados mediante la prueba $t$ de Student para muestras independientes (cuando se trató de ver si existían diferencias entre nativos e inmigrantes). A la hora de la interpretación de los resultados, se tuvo en cuenta la existencia, o no, de homogeneidad de varianzas.

Como medida del tamaño del efecto se ha utilizado el coeficiente eta-cuadrado parcial $\left(\eta_{\mathrm{p}}{ }^{2}\right)$, ya que es uno de los procedimientos más utilizados habitualmente dentro de la investigación educativa (Sun, Pan y Wang, 2010). Para la interpretación de los tamaños del efecto se utiliza el criterio establecido en el trabajo clásico de Cohen (1988), en base al cual, un efecto es pequeño cuando $\eta_{p}{ }^{2}=$ $.01(d=.20)$, el efecto es medio cuando $\eta_{p}{ }^{2}$ $=.059(d=.50)$ y el tamaño del efecto es grande si $\eta_{p}{ }^{2}=.138(d=.80)$.

\section{RESULTADOS}

En la Tabla 1 se aportan los estadísticos descriptivos correspondientes. En general, los resultados de los análisis de diferencias de medias indican que mientras que en alguna variable no se observan diferencias estadísticamente significativas, en otras sí hay diferencias entre ambos grupos de estudiantes. Seguidamente se exponen los resultados por variable.

En la cantidad de deberes realizados (de los prescritos), los datos obtenidos indican que los alumnos no informan de diferencias estadísticamente significativas entre ellos en cuanto a esta variable $(t(456)=-0.924 ; p>$ $.05, d=.09$, tamaño del efecto pequeño).

En lo que respecta al tiempo dedicado a la realización de deberes (semanal), como se puede observar en la Tabla 1, los propios alumnos nativos dedican más tiempo a la realización de los deberes escolares, pero esta diferencia no es estadísticamente significativa $(t(499)=1.241, p>.05, d=.86$, tamaño del efecto grande).

Para el aprovechamiento del tiempo (dedicado a los deberes), según se desprende de los datos aportados en la Tabla 1, los estudiantes nativos manifiestan que aprovechan mejor que los inmigrantes el tiempo que dedican a la realización de los deberes escolares $(t(498)=5.035, p<.001)$.

En lo referido a la calidad de los deberes realizados, los profesores indican que los estudiantes nativos llevan al colegio sus deberes realizados con una mayor calidad que los estudiantes inmigrantes $(t(477)=$ 2.109, $p<.05, d=.19$, tamaño del efecto pequeño).

Según la variable repercusiones (de la realización de los deberes), también los profesores creen que la realización de deberes beneficia más a los estudiantes nativos que a los inmigrantes $(+(375)=2.747, p<.05, d=$ .28, tamaño del efecto pequeño).

Finalmente, respecto del rendimiento académico se observan diferencias estadísticamente significativas en cuanto al rendimiento académico $(t(497)=8.879, p<$ $.001, d=.81$, tamaño del efecto grande), obteniendo un mayor rendimiento los estudiantes nativos.

\section{DISCUSIÓN}

Dado que en la investigación previa (Bang, Suárez-Orozco, Pakes, \& O'Connor, 2009) se aportan datos poco exactos y muy generales sobre el tema de los deberes escolares en alumnado inmigrante el objetivo del presente estudio es comprobar si la relación entre la implicación del alumno (medida por la cantidad de deberes que realizan de los prescritos por los profesores, la cantidad de tiempo dedicada a los mismos y la gestión de ese tiempo entendida como aprovechamiento del tiempo invertido) y el rendimiento académico es diferente entre población nativa e inmigrante.

Según los hallazgos, los alumnos nativos hacen deberes de más calidad, se benefician 
Tabla 7

Estadísticos descriptivos de los grupos de estudiantes nativos e inmigrantes

\begin{tabular}{|c|c|c|c|c|}
\hline & Grupo & $\mathrm{n}$ & M & $\mathrm{DE}$ \\
\hline \multirow[t]{2}{*}{ Alumno. ¿Cuántos deberes haces? } & Nativo & 310 & 4.16 & 1.068 \\
\hline & Inmigrante & 191 & 4.25 & 0.887 \\
\hline \multirow[t]{2}{*}{ Alumno. Tiempo dedicado cada día de lunes a viernes } & Nativo & 310 & 3.10 & 1.155 \\
\hline & Inmigrante & 191 & 2.97 & 1.147 \\
\hline \multirow[t]{2}{*}{ Alumno. Al hacer los deberes, me concentro hasta que termino } & Nativo & 310 & 3.11 & 1.132 \\
\hline & Inmigrante & 190 & 2.59 & 1.103 \\
\hline \multirow[t]{2}{*}{ Profesores. ¿Cómo trae los deberes hechos? } & Nativo & 288 & 3.74 & 1.153 \\
\hline & Inmigrante & 191 & 3.51 & 1.213 \\
\hline \multirow[t]{2}{*}{ Profesores. ¿Se beneficia de realizar los deberes? } & Nativo & 288 & 3.97 & 1.107 \\
\hline & Inmigrante & 191 & 3.66 & 1.137 \\
\hline \multirow[t]{2}{*}{ Nota de Matemáticas Secundaria } & Nativo & 309 & 6.10 & 1.945 \\
\hline & Inmigrante & 190 & 4.51 & 1.946 \\
\hline
\end{tabular}

más de su realización, aprovechan mejor el tiempo y obtienen un mayor rendimiento que sus compañeros inmigrantes. En la variable gestión del tiempo se han encontrado diferencias: los estudiantes nativos manifiestan que se concentran más que los inmigrantes en la realización de los deberes escolares.

A modo de síntesis podríamos decir que aunque el hecho de realizar los deberes escolares resulta beneficioso para el alumnado inmigrante, las dos poblaciones presentan diferencias en las variables que miden la implicación escolar. Éstas variables guardan relación con el rendimiento académico tal y como se ha visto en publicaciones anteriores (Cooper, 1989; Núñez et al., 2013). Las diferencias encontradas nos hacen sospechar que quizás existan otros factores que puedan influir en la implicación del estudiante como apuntaban otros investigadores (Bang, Suárez-Orozco, Pakes, \& O’Connor, 2011 ).

Por todo ello, resulta de interés de cara a futuras investigaciones ahondar en nuevas variables que puedan dar cuenta de la implicación de estos alumnos de una manera más precisa. Ya que parece que aunque estos criterios si se relacionan con el rendimiento, al existir diferencias con el alumnado nativo, quizás pueda existir algún otro criterio que nos ayude a explicar la variabilidad en los resultados.

Se han apuntado diferentes variables que pueden afectar a la implicación: el dominio del idioma; la cultura de procedencia (Mau \& Lynn, 1999); la ayuda ofrecida por los padres
(Madjar, Shklar, \& Moshe, 2016; Núñez, Suárez, Rosário, Vallejo, Valle, et al., 2015); el feedback dado por el profesor (Núñez, Suárez, Rosário, Vallejo, Cerezo, et al., 2015). Si bien es cierto que quizás también haya que ahondar en el tipo de deberes que se pone a los alumnos, ya que no cualquier tipo de tarea resulta igual de efectiva de cara a obtener un buen rendimiento académico.

Cabe destacar que por las características del estudio no tenemos certeza de la dirección de la relación; es decir, si una mayor implicación es la causante de un mayor rendimiento académico, mejores resultados académicos llevan a que el alumno se implique más o, ambas direcciones tienen un peso importante en esta relación (Sonnenschein \& Galindo, 2015). Aunque en la literatura previa con población nativa se ha propuesto un modelo en el que se apunta por la primera dirección (Núñez et al., 2013).

Quizás en este estudio se echa en falta encontrar un modelo predictivo con el que pronosticar la varianza explicada del constructo total, en el que se tomase la calidad de la tarea realizada como una variable moduladora entre la relación entre implicación y rendimiento, dada la correlación positiva y significativa encontrada entre esta última variable y el rendimiento académico. Sin embargo, dadas las características de los datos recogidos no ha sido posible realizarlo. Otra limitación de nuestro estudio ha sido la selección de la muestra, ya que dada la premura del tiempo y las características de 
la población a estudiar nos ha sido difícil encontrar participantes para la recogida de datos y, por tanto, dada la escasez de alumnado con estas características al que teníamos acceso ha tenido que realizarse de este modo, sin aleatorizar.

\section{Conflicto de intereses}

Los autores declaran no tener ningún conflicto de intereses.

\section{Agradecimientos}

Este trabajo se ha desarrollado gracias a la financiación de los proyectos de investigación EDU2013-44062-P (MINECO) y EDU2017-82984-P (MEIC).

\section{REFERENCIAS}

Bang, H. J., Suárez-Orozco C., Pakes J., \& O'Connor E. (2009). The importance of homework in determining immigrant students' grades in schools in the USA context. The Journal of Educational Research, 51(1), 1-25. doi:10.1080/00131880802704624

Cohen, J. (1988). Statistical power analysis for the behavioral sciences ( $2^{a}$ ed.). Hillsdale, NJ: Erlbaum.

Cooper, H. (1989). Homework. New York: Longman.

Cooper, H. (2007). The battle over homework (3rd ed.). Thousand Oaks, CA: Corwin Press.

Cooper, H., Robinson, J., \& Patall, E. (2006). Does homework improve academic achievement? A synthesis of research, 1987 2003. Review of Educational Research, 76, $1-62$.

De Jong, R., Westerhof, K. J., \& Creemers, B. P. M. (2000). Homework and student math achievement in junior high schools. Educational Research and Evaluation, 6, 130-157. doi:10.1076/1380$3611(200006) 6: 2 ; 1-E ; F 130$

Dettmers, S., Lüdtke, O., Trautwein, U., Kunter, M., \& Baumert, J. (2010). Homework works if homework quality is high: Using multilevel modeling to predict the development of achievement in mathematics. Journal of Educational Psychology, 102(2), 467-482. doi:10.1037/a0018453
Ibáñez, I. (2012). Los deberes escolares: Un poderoso aliado para el aprendizaje y la formación. Universo UP, 18. Retrieved from http://revista.universidaddepadres. es /index.php?option=com_content\&view $=$ article\&id $=1456$

Madjar, N., Shklar, N., \& Moshe, L. (2016). The role of parental attitudes in children's motivation toward homework assignments. Psychology in the Schools, 53(2), 173-188. doi: 10.1002/pits.21890

Mau, W., \& Lynn, R. (1999). Racial and ethnic differences in motivation for educational achievement in the United States. Personality and Individual Differences, 27(6), 10911096.

Muhlenbruck, L., Cooper, H., Nye, B., \& Lindsay, J. J. (2000). Homework and achievement: explaining the different strengths of relation at the elementary and secondary school levels. Social Psychology of Education, 3, 295-317. doi:10.1023/A:1009680513901

Núñez, J. C., Suárez, N., Rosário, P., Vallejo, G., Cerezo, R., \& Valle, A. (2015). Teachers' feedback on homework, homework-related behaviors and academic achievement. The Journal of Educational Research, 108, 204216. doi:10.1080/00220671.2013.8782 98

Núñez, J. C., Suárez, N., Rosário, P., Vallejo, G., Valle, A., \& Epstein, J. L. (2015). Relationships between parental involvement in homework, student homework behaviors, and academic achievement: Differences among elementary, junior high, and high school students. Metacognition and Learning. 10, 375-406. doi:10.1007/s1 1409-0159135-5

Núñez, J.C., Suárez, N., Cerezo, R., GonzálezPienda, J.A., Rosário, P., Mourão, R., \& Valle, A. (2013). Homework and academic achievement across Spanish Compulsory, Education, Educational Psychology. 35(6), 726-746.

Sonnenschein, S., \& Galindo, C. (2015). Race / ethnicity and early mathematics skills: relations between home, classroom, and mathematics achievement. The Journal of 
Educational Research, 108, 261-277. doi:1 $0.1080 / 00220671.2014 .880394$

Sun, Sh., Pan, W., y Wang, L. L. (2010). A comprehensive review of effect size reporting and interpreting practices in academic journals in education and psychology. Journal of Educational Psychology, 102(4), 989-1004. doi: 10.1037/a0019507

Tam, V. C. (2009). Homework involvement among Hong Kong primary school students. Asian Pacific Journal of Education, 29, 213227. doi:10.1080/02188790902859004

Trautwein, U., \& Lüdtke, O. (2009). Predicting homework motivation and homework effort in six school subjects: The role of person and family caracteristics, classroom factors and school track. Learning and Instruction, 19, 243-258. doi:10.1016/i. learninstruc.2008.05.001

Trautwein, U., Lüdtke, O., Schnyder, I., \& Niggli, A. (2006). Predicting homework effort: Support for a domain-specific, multilevel homework model. Journal of Educational Psychology, 98(2), 438-456. doi:10.1037/0022-0663.98.2.438

Trautwein, U., Schnyder, I., Niggli, A., Neumann, M., \& Lüdtke, O. (2009). Chameleon effects in homework research: The homeworkachievement association depends on the measures used and the level of analysis chosen. Contemporary Educational Psychology, 34(1), 77-88. doi:10.1016/i. cedpsych.2008.09.001

Vila, D. (2012). A vueltas con los deberes. Revista electrónica de la mutualidad general de funcionarios civiles del estado (222//2012). Retrieved from http://www. muface.es/revista/i222/educacion_vuelta deberes.html

Xu, J. (2007). Middle-School Homework Management: More than just gender and family involvement. Educational Psychology, 27(2), 173-189. doi:10.1080/01443410601066669

Xu, J. (2008). Models of secondary school students' interest in homework: A multilevel analysis. American Educational Research Journal, 45, 1180-1205. doi: $10.3102 / 0002831208323276$

Xu, J. (2010). Predicting homework time management at the secondary school level: A multilevel analysis. Learning and Individual Differences, 20, 34-39. doi:10.1016/i. lindif.2009.11.001

Zimmerman, B. J., \& Kitsantas, A. (2005). Students' perceived responsibility and completion of homework: The role of self-regulatory beliefs and processes. Contemporary Educational Psychology, 30, 397-417. 